\title{
Acute Biphenotypic Leukemia
}

National Cancer Institute

\section{Source}

National Cancer Institute. Acute Biphenotypic Leukemia. NCI Thesaurus. Code C4673.

An acute leukemia of ambiguous lineage characterized by blasts which coexpress

myeloid and T or B lineage antigens or concurrent B and T lineage antigens. (WHO,

2001) 